\title{
Circumstances, Patterns, and Reporting of Incidents of Workplace Violence among Resident Doctors Posted at Selected Departments of a Tertiary-Care Hospital in Kolkata, India
}

\author{
Mousumi Datta, M.D., Adwitiya Das, M.D.
}

Department of Community Medicine, Medical College Kolkata, West Bengal University of Health Sciences, Kolkata, West Bengal 700073, India.

Received 31 October 2020 • Revised 11 February 2021 • Accepted 23 February 2021 • Published online 31 May 2021

\begin{abstract}
:
Objective: Violence at work is a major cause of workplace inequality, discrimination, stigmatization, and conflict. The present study intends to describe the pattern of workplace violence (WPV) in a tertiary-level hospital in India with the objectives of discovering the magnitude of self-reported violence, its type, and the circumstances surrounding and response to such incidents.

Material and Methods: The study is of a descriptive type. Its participants were either undergraduate or postgraduate residents at the selected hospital. The data collection tool was and a questionnaire adapted from that used in the World Health Organization's Workplace Violence in the Health Sector Survey. The period of data collection was one month.

Results: Three hundred and twenty two completed questionnaires were analyzed; $76.1 \%$ of respondents experienced violence at their workplace. The incidents of psychological violence were higher than those of a physical nature $(63.1 \%$ vs. $42.9 \%$, respectively). Psychological violence was significantly higher during day shifts and in outdoor or emergency room settings, while physical violence was common during night shifts and in indoor wards. Repeat incidents were more commonly associated with psychological violence. Even though most incidents were not reported, 96.0\% of respondents considered WPV a major concern, and the improvement of security measures was most widely suggested as a solution to prevent violence at work.
\end{abstract}

Conclusion: The high incidence of WPV in a tertiary healthcare setting reported in this study is in agreement with the findings of similar studies. Improving security measures and doctor- patient communication may help reduce such

Contact: Asst. Prof. Mousumi Datta, M.D.

Department of Community Medicine, Medical College Kolkata,

West Bengal University of Health Sciences, Kolkata, West Bengal 700073, India.

E-mail: drmousumid@gmail.com

This is an open access article under the CC BY-NC-ND license

(http://www.jhsmr.org/index.php/jhsmr/about/editorialPolicies\#openAccessPolicy).

J Health Sci Med Res 2022;40(1):35-43 doi: 10.31584 /jhsmr.2021815 www.jhsmr.org 
occurrences. Designing a robust reporting system and conducting regular and thorough audits of violent incidents can help boost the morale of healthcare staffs as well as plan preventive strategies.

Keywords: doctors, hospitals, India, workplace violence

\section{Introduction}

Workplace violence (WPV) is defined as incidents where staffs are abused, threatened or assaulted in circumstances related to their work, including commuting to and from work, involving an explicit or implicit challenge to their safety, wellbeing, and health. ${ }^{1}$ The National Institute of Occupational Safety and Health categorises WPV into four types, of which WPV in the healthcare sector usually belongs to Type II (aggressor being recipient of service) or Type III violence (aggressor being another employee, supervisor, or manager). It can also be categorised as physical or non-physical violence. ${ }^{2}$

Violence at work affects the dignity of millions of healthcare professionals. It is a major cause of inequality, discrimination, stigmatization, and conflict at workplace. ${ }^{3}$ The third European study on working conditions stated that violence at work clearly leads to an increase in health complaints, particularly stress. Being a victim of WPV can lead to a host of adverse psychological outcomes ranging from shock, anger, and disbelief to increased stress level, loss of job satisfaction, and even self-harm. ${ }^{4}$ Sickness absenteeism is found to be $26.0 \%$ higher among bullied hospital workers with over a quarter of them ending up leaving work. ${ }^{5}$ This turnover leads to the loss of knowledge, skills, and training investment followed by the consequent costs of hiring new staffs. This may result in pan-sector personnel recruitment and retention difficulties.

WPV-related serious injury (requiring days off) in the healthcare industry is about four times higher than those related to other private sectors. ${ }^{6}$ In Western countries, patients are the perpetrators in about $80.0 \%$ of violent incident cases. The implicated risk factors for WPV are working with people with a history of violence or under the influence of drugs or alcohol, transporting patients, delivering direct patient care, poor design of workplace environment, working alone, lack of emergency communication, lack of worker training, inadequate security staffs, long patient waiting time, unrestricted public access to healthcare facilities, and the perception that violence is tolerable and reporting incidents of violence will have no positive effect. ${ }^{6}$ There is, however, no conclusive evidence to link WPV with perpetrator or victim demographics, or urban versus suburban or rural workplace settings. ${ }^{7,8}$

The underreporting of cases of WPV is a recurrent observation. Only $30.0 \%$ of nurses report incidents of WPV; among emergency department physicians, the reporting rate is even lower (26.0\%). ${ }^{9,10}$ Underreporting leads to the underestimation of the extent of the problem and hinders the effective planning of violence-control strategies. Moreover, a study from the US found that $88.0 \%$ of self-reported incidents of WPV were not formally reported using an electronic system. ${ }^{11}$

The Joint Commission urges health personnel to remain alert about violence at workplace and health employers to look beyond security solutions as an answer to WPV. It has also published a comprehensive evidencebased recommendation to cope with WPV. ${ }^{12}$

A review of 12 WPV-prevention training programs, developed from training topics of Occupational Safety and Health Agency guidelines for preventing WPV for healthcare and social service workers, showed that none of the training programs addressed all the reviewed criteria. 
The most significant gap involved the lack of attention paid to facility-specific risk assessment and policies. ${ }^{13}$

As evident from the above discussion, WPV is the result of a complex interplay between factors related to workers, clients, and organizations; compared to their Western counterparts, the ones in India differ significantly. India has the largest number of medical colleges in the world, with an annual output of $>30,000$ medical graduates and $>18,000$ specialist doctors of modern medicine. ${ }^{14}$ Although India meets the World Health Organization's (WHO) standard for the health sector manpower of one doctor for every 1,000 population, considerable regional disparities exists. Indian doctors often work under highly challenging circumstances, which contributes to a heightened risk for violence. A 2018 study analyzed the incidents of mediareported violence in Indian hospitals that led to doctors leaving their jobs. The results showed that such incidents have been on the rise since 2013. Furthermore, in that study, $41.0 \%$ of incidents resulted in grievous hurt and $2.0 \%$ in deaths. ${ }^{15}$ Another multi-centric study from Uttar Pradesh, Northern India reported a $87.0 \%$ exposure rate to WPV among health workers over a 12-month period. ${ }^{16}$ Despite such an alarming scenario, violence-prevention training among Indian doctors is lacking; 90.4\% report having had no such formal training. ${ }^{17}$ Moreover, training related to violence reporting and proper organizational response to violence is often neglected; one study reported that $61.3 \%$ of respondents were not aware of any violence-prevention policy at their workplace. ${ }^{18}$

Ultimately violence costs the victim, the perpetrator, and the state as well as the insurance systems and the people who fund it. Organizations bear the costs of WPV in terms of time off work taken by victims, temporary staff cover, and fees for legal action, medical treatment, and counselling. In addition, hospital services can suffer due to worker protests against incidents of violence, which leads to the inability of patients to utilize healthcare services. Furthermore, the safety and security of workers at their workplace is a matter of human rights and must be a priority for employers. In light of the above-mentioned, this study was conducted with the objectives of assessing the magnitude and types of self-reported violence among the resident doctors of an Indian teaching hospital as well as the circumstances surrounding such incidents of violence and the institutional response to them.

\section{Material and Methods}

The present study was of a descriptive type employing a cross-sectional design. All undergraduate and postgraduate trainee doctors working at Kolkata Medical College Hospital were eligible to participate in the study. The inclusion criterion was being a trainee doctor with a work history of last six months, who consented to participate in the study; trainees posted in departments that were not covered for data collection were automatically excluded. Stat Cal version 4.0 was used for sample size calculation. The confidence interval was set at $95.0 \%$, the total population size was 1,200 , i.e., the total number of undergraduate (UG) and postgraduate $(\mathrm{PG})$ trainees, and the a priori incidence of WPV was $47.0 \% .{ }^{17}$ With a maximum acceptable difference of $5.0 \%$, the minimum sample size was estimated to be 278. By discussion with competent authorities, it was found that the majority of incidents of WPV occurred in the Departments of Medicine, Surgery, Gynecology and Obstetrics, Pediatrics, Orthopedics, and Emergency. Hence, these departments were selected for data collection, and doctors working in these departments during the study period were contacted. The expected sample size was rounded up to 360 presuming a $10.0 \%$ rate of missing data and to facilitate the drawing of samples from the six departments of interest. Sixty doctors were randomly selected from each department. Lists of UG and PG trainees posted during the study period were made for each department. The participants were selected via a random number table using their serial number on those lists. Separate lists were used for UG and PG trainees, and 
they were recruited in a 1:1 ratio; however, the Emergency Department had a much higher number of UG residents compared to the other departments. The complicated duty rosters including days off, repeat duties, multiple shifts, and duty rotation were given due care during data collection. Those who refused to participate were replaced by another trainee doctor of the same academic rank and posted at the same department.

Data Collection: A predesigned, pretested, semistructured, self-administered questionnaire adapted and validated from the WHO's Workplace Violence in the Health Sector Survey questionnaire served as the tool for data collection. ${ }^{18}$ The data were collected over one month during the July-August 2019 period.

Variables: The core variables were the magnitude and type of violent incidents occurring within the previous six months, the place and time of their occurrence, whether they were reported or not, and what actions were taken as a consequence. The demographic variables were sex, category of trainee, and mother tongue; the occupational variables were average hours of work per week, number of co-workers per shift, and the respondent's perception related to WPV.

Operational definitions of core variables: These definitions were modified from those provided in the study tool. Physical violence was defined as any degree of intentional physical harm by another person, including those of a sexual nature. This included pushing, beating, slapping, pinching, biting, inappropriate touching, and incidents of a similar nature occurring at one's workplace. ${ }^{18}$ Psychological violence was defined as the intentional use of power to humiliate another person. This included verbal abuse, bullying, harassment, threats, and incidents of a similar nature occurring at or related to one's workplace. ${ }^{18}$

Statistical analysis: Frequencies and proportions were calculated to describe categorical variables. The $Z$ test for the difference of two proportions was the statistical test of choice to determine statistical significance. Microsoft
Excel and Statistical Package for the Social Science for Windows (SPSS) version 17 were used for all data entry and calculations.

Ethical consideration: Ethical approval for the study was obtained from the Institutional Ethics Committee of the Medical College Kolkata (approval number MC/KOL/EC/ NON-SPON/447/08/19). All study participants provided an informed consent.

\section{Results}

The study results are based on the analysis of 322 completed questionnaires. The mean age of the respondents was $25.3( \pm 2.7)$ yrs.; $64.3 \%$ were male, and their mean duration of work experience was of $8.5( \pm 2.3)$ months. The majority of respondents were junior residents (interns) and fluent in the local vernacular, Bengali. Most participants $(75.2 \%)$ worked $>48$ hrs. per week, and all of them did shift duties during both shifts (day and night shifts). Only a small proportion of respondents reported working alone during their shifts; most had between one and five co-workers working with them (Table 1). Of the total, 245 (76.1\%) respondents reported having ever experienced violence at their workplace (one or more episodes of violence in the last six months of the physical, psychological, or both types). Regarding the type of violence, 138 (42.9\%) had experienced physical violence, while 203 (63.1\%) had suffered psychological violence. Ninety-four (29.2\%) respondents had experienced both forms of violence. Compared to physical violence, the psychological violence incidents were significantly higher $(Z=5.00, p$-value<0.00). In relation to psychological violence, $82.7 \%$ of the incidents involved verbal abuse. Psychological violence was more likely to occur during day shifts (from 8 am to $3 \mathrm{pm}$ ), at outpatient departments, and at the Emergency Department. Physical violence occurred in significantly higher proportions during night shifts (from $3 \mathrm{pm}$ to $8 \mathrm{am}$ ) and in indoor wards. Nearly all respondents $(96.9 \%)$ perceived violence at work to be a serious threat. Most $(54.0 \%)$ of them were 
not aware of any violence-prevention policies at their workplace. Violent incidents were formally reported by $41.7 \%$ of respondents. Although the overall reporting rate was low, it was slightly higher for incidents involving physical violence. According to the respondents, action was not taken for most reported incidents, and by and large, the respondents were dissatisfied with the organizational response to WPV. It was also found that $94.2 \%$ of respondents who experienced physical violence and $86.2 \%$ of respondents who faced psychological violence considered those events as preventable. Table 4 records the data related to the perception of respondents about drivers of violence and possible preventive measures. Multiple responses were allowed. Lack of adequate security was widely suggested as a major driver for incidents of violence, and almost every respondent suggested improving security at hospitals as the solution to curbing such incidents. 'Poor health literacy' and 'unreasonable expectations for a magic cure' were also important drivers for violence according to the respondents.

Table 1 Distribution of respondents according to their demography and work-related circumstances $(n=322)$

\begin{tabular}{lll}
\hline Variables & Categories & Number (\%) \\
\hline Sex & Male & $207(64.3)$ \\
& Female & $115(35.7)$ \\
Category of trainee & Undergraduate trainee & $224(69.6)$ \\
& Postgraduate trainee & $98(30.4)$ \\
Mother tongue & Bengali & $264(82.0)$ \\
& Others & $58(18.0)$ \\
Average hours of work & $\leq 48$ & $80(24.8)$ \\
per week & $>48-72$ & $242(75.2)$ \\
No. of coworkers & None & $4(1.2)$ \\
during day shift & $1-5$ & $245(76.1)$ \\
& $\geq 6$ & $73(22.7)$ \\
No. of coworkers & None & $24(7.5)$ \\
during night shift & $1-5$ & $266(82.6)$ \\
& $\geq 6$ & $32(9.9)$ \\
\hline
\end{tabular}

Table 2 Distribution of respondents according to their experiences of workplace physical and psychological violence over the previous six months

\begin{tabular}{|c|c|c|c|}
\hline \multirow{2}{*}{ Variables } & \multicolumn{2}{|c|}{ Type of violence } & \multirow{2}{*}{ Significance } \\
\hline & Physical (n=138) & Psychological $(n=203)$ & \\
\hline Incidents of violence being repeat ones & $79(57.2)$ & $153(75.4)$ & $\begin{array}{l}Z=3.3 \\
p-\text { value }=0.001^{*}\end{array}$ \\
\hline $\begin{array}{l}\text { Perception of incidents of violence being typical in one's } \\
\text { workplace setting } \\
\text { Shift of occurrence \# }\end{array}$ & $104(75.6)$ & $159(78.3)$ & $\begin{array}{l}Z=0.5 \\
p-\text { value }=0.608\end{array}$ \\
\hline Day & $70(50.77)$ & $161(79.3)$ & $\begin{array}{l}Z=5.4 \\
p-\text { value }=0.000^{*}\end{array}$ \\
\hline Night & $68(49.3)$ & $42(20.7)$ & $\begin{array}{l}Z=5.5 \\
p-\text { value }=0.000^{*}\end{array}$ \\
\hline \multicolumn{4}{|l|}{ Place of occurrence \# } \\
\hline Outdoor/emergency setting & $28(20.3)$ & $100(49.3)$ & $\begin{array}{l}Z=5.3 \\
p-\text { value }=0.000^{*}\end{array}$ \\
\hline Indoor setting & $110(79.7)$ & $103(50.7)$ & $\begin{array}{l}Z=5.1 \\
p-\text { value }=0.000^{*}\end{array}$ \\
\hline \multicolumn{4}{|l|}{ Time of week \# } \\
\hline Weekdays & $92(66.6)$ & $154(75.9)$ & $\begin{array}{l}Z=1.6 \\
p-\text { value }=0.092\end{array}$ \\
\hline Weekends & $46(33.4)$ & $49(24.1)$ & $\begin{array}{l}Z=1.7 \\
p-\text { value }=0.084\end{array}$ \\
\hline
\end{tabular}

\#Experience of last incident of violence 
Table 3 Distribution of respondents according to their perception of and response to workplace violence

\begin{tabular}{|c|c|c|}
\hline Variables & Number (\%) & \\
\hline Perception of violence being a serious threat at one's workplace & $312 / 322(96.9)$ & \\
\hline Awareness of any institutional policy related to workplace violence & $148 / 322(46.0)$ & \\
\hline $\begin{array}{l}\text { Formal report of an incident of workplace violence experienced during the previous six } \\
\text { months to authorities/police }\end{array}$ & $102 / 245$ (41.6) & \\
\hline Reporting of WPV and action taken according to type of violence & Physical violence & Psychological violence \\
\hline Incidents reported & $55 / 138$ (39.8) & $47 / 203(23.1)$ \\
\hline Action taken for reported incidents & $16 / 55(29.1)$ & $9 / 47(19.1)$ \\
\hline Satisfied with organizational response & 2/55 (3.6) & $2 / 47(4.2)$ \\
\hline
\end{tabular}

Table 4 Respondent perceptions regarding drivers of workplace violence and possible measures to prevent it ( $n=322$ )

\begin{tabular}{|c|c|}
\hline Drivers behind incidents of violence & Percentage \\
\hline Poor health literacy/unreasonable expectation among patients & 30.4 \\
\hline Unrestricted entry of visitors to care facilities/inadequate security & 28.2 \\
\hline Inadequate communication & 20.8 \\
\hline Long patient waiting times/complicated care protocols & 15.8 \\
\hline Aggressive/armed/drunk visitors & 15.2 \\
\hline Inadequate number of staffs & 11.8 \\
\hline Political interference/illegal exchange of money for healthcare services & 6.2 \\
\hline Patient health condition (death/sudden deterioration) & 3.1 \\
\hline Lack of strict laws against violence & 2.4 \\
\hline Negative image of doctors created by media & 4.6 \\
\hline Suggested measures to prevent violence & Percentage \\
\hline Improve security measures & 91.6 \\
\hline Restrict public access to care facilities & 71.1 \\
\hline Improve surroundings of care facilities & 65.5 \\
\hline Increase the number of hospital staff & 65.5 \\
\hline Improve patient screening & 49.4 \\
\hline Provide better staff training & 41.9 \\
\hline Provide adequate staff uniforms & 36.3 \\
\hline Improve patient-care protocols & 35.4 \\
\hline Provide a system for the rapid reporting of incidents of violence & 32.9 \\
\hline
\end{tabular}

\section{Discussion}

Our study reports a high burden of WPV; $76.1 \%$ of respondents reported having experienced WPV during their work tenure of the last six months. Singh et al. in their multicentric study from Uttar Pradesh found a $69.5 \%$ incidence rate of WPV. ${ }^{16}$ Reported experiences of WPV vary from $40.8-47.0 \%$ in studies from Delhi to $78.2 \%$ in a study from
Manipur. ${ }^{17,19,20,21}$ A meta-analysis of WPV against healthcare professionals in China, meanwhile, reported a prevalence of $62.4 \%(\mathrm{Cl} 59.4 \%$ to $65.5 \%) .{ }^{22}$ Differing institutional and human factors between study settings can be attributed for this variation in WPV magnitude. Nevertheless, as seen in the two Delhi reports, studies from the same geographical area tend to show similar prevalence rates; it is probable, 
therefore, that both local culture and the perceived image of health workers play a role. ${ }^{17,20}$ Psychological violence was the more common type of WPV in our study, of which the highest incidence was associated with verbal abuse. This pattern is congruent with the findings of many other studies. ${ }^{16,20,22}$ While physical violence can result in serious injuries and even fatalities, non-physical violence is more covert. Psychological or non-physical violence is the more common WPV type of the two, yet it is much underreported. In this study, such violence was reported being frequent, repeated, and taking place in crowded outpatient departments, which points out the fact that it takes little for people to abuse or bully a healthcare staff. Strong links have been found between the incidence of physical violence and one's intention to quit the workplace or profession. ${ }^{23,24}$

Security-related issues were considered major drivers of violence by the participants, and improving security measures was also the most often suggested solution to prevent WPV. Improving security is an obvious and common-sense recommendation to deal with violence. The European Agency for Safety and Health at Work recommends preventive measures like increased security presence, liaison with police, and paying attention to ergonomics with a view to prevent violence and ensure a safe work practice. Panic alarms, television coverage, and disclosure regarding policies related to WPV should also be present. ${ }^{25}$ Roughly $79.0 \%$ of physical and $50.0 \%$ of psychological violence incidents in our study happened indoors, which indicates a poor security plan at the hospital that was our study setting. Moreover, acts of violence are primed by a negative image of doctors perpetuated by media, political interference, and the illegal solicitation of finances for medical services by unscrupulous hospital staffs. A qualitative study conducted in Norway broadly aligns with the themes of concern raised by the respondents in our study, namely security issues (working alone), lack of training to deal with incidents of violence (unpreparedness), mismatch between patient/visitor expectations and realistic deliverables befitting the care setting, and inadequate organisational response to WPV (managerial support). ${ }^{26}$

Nevertheless, medical personnel themselves can do a lot to prevent violence. Our respondents widely cited inadequate doctor-patient communication as a driver of violence in the care setting. Focus group discussions involving hospital staffs from Israel helped identify themes related to both patient as well as staff behaviour and expectations, which can result in conflicts. ${ }^{27}$ Staff training on communication and de-escalation techniques was mentioned as a preventive measure by $>40.0 \%$ of respondents in the present study. It can also be appreciated that security measures can often do little to prevent nonphysical violence. Moreover, there was no formal violenceprevention training offered at the institution under study. Patients often became frustrated by the inadequacies related to the amount of medical personnel, long waiting times, and complicated protocols for service delivery, which provide the necessary spark for violence to occur. Beside improving the quality of service, managing patient queues and staff rosters efficiently, making service protocols more client-centred, and providing proper signage throughout the care facilities are small measures that can be taken by the management, which can go a long way toward preventing WPV.

Our review of literature on evidence-based interventions to prevent WPV emphasised underreporting as a deterrent to prevention. ${ }^{28}$ We also found a low reporting rate of incidents of violence. In our study, the victims might have decided to not report the incidents of violence perpetrated on them due to the fact that, in most such situations, no action is taken. There was also no formal digital or physical system to report incidents of violence. It is quite evident that an effective information system and surveillance program to combat WPV are the needs of the hour for this care institution. 
A strength of this study is the use of a standardised tool in a region where despite the high prevalence of WPV in the health sector, this topic remains largely unexplored. The scope of this study could be widened further by including more categories of healthcare workers like nursing staffs, patient attendants, and even community-level health workers or different levels of healthcare institutions. Moreover, an in-depth study of the epidemiology of non-physiological violence in the health sector of India is warranted. The managerial aspects and economic impact of implementing WPV-prevention strategies also need to be studied. Finally, this survey has the limitations of being a single-centre study and employing self-reported data.

\section{Conclusion}

The high incidence of WPV in a tertiary healthcare setting reported in this study is in agreement with the findings reported by similar studies. To prevent incidents of violence at work, effective policies and strategies aimed at combating this phenomenon must be embedded in the culture of organisations. They should include both security as well as non-security measures. In particular, improving security measures and doctor-patient communication may help reduce incidents of WPV. Furthermore, staffs should be encouraged to always report incidents of violence. Finally, designing and implementing robust reporting systems and conducting thorough investigations of incidents of violence would be very beneficial towards both boosting the morale of health workers and planning effective preventive strategies.

\section{Conflict of interest}

None

\section{References}

1. ILO, ICN, WHO. Framework guidelines for addressing workplace violence in the health sector, Joint programme on work place violence in health sector. Geneva: Publications Bureau International Labour Office; 2002.
2. Martino V. ILO, ICN, WHO. Workplace violence in health sector. Country case studies Brazil, Bulgaria, Lebanon, Portugal, South Africa, Thailand and an additional Australian study Synthesis report. Workplace violence in the health sector. Geneva: Publications Bureau International Labour Office; 2002.

3. Nordin H. Fakta om vaold och hot I arbetet, Solna, Occupational Injury Information System. Solna: Swedish Board of Occupational Safety and Health; 1995.

4. International Council of Nurses. Guidelines on coping with violence in the workplace. Geneva: Publications Bureau International Labour Office; 1999.

5. Kivimaki K, Elovainio M, Vathera J. Workplace Bullying and sickness absence in hospital staff. Occup Environ Med 2000;57:656-60.

6. Occupational Safety and Health Administration. Guidelines for preventing workplace violence for healthcare and social service workers. Washington DC: OSHA; 2015.

7. Gates D, Gillespie G, Kowalenko T, Succop P, Sanker M, Farra S. Occupational and demographic factors associated with violence in the emergency department. Adv Emerg Nurs $\mathrm{J}$ 2011;33:303-13.

8. Kowalenko T, Gates D, Gillespie G, Succop P, Mentzel TK. Prospective study of violence against ED workers. Am J Emerg Med 2013;31:197-205.

9. Speroni KG, Fitch T, Dawson E, Dugan L, Atherton M. Incidence and cost of nurse workplace violence perpetrated by hospital patients or patient visitors. J Emerg Nurs 2014;40: 218-28.

10. Behnam M, Tillotson RD, Davis SM, Hobbs GR. Violence in the emergency department: A national survey of emergency medicine residents and attending physicians. J Emerg Med 2011;40:565-79.

11. Arnetz JE, Hamblin L, Ager J, Luborsky M, Upfal MJ, Russel $J$, et al. Underreporting of workplace violence. Comparison of self-report and actual documentation of hospital incidents. Workplace Health Saf 2015;63:200-10.

12. The Joint Commission. Physical and verbal violence against health care workers. Sentinel Event Alert 2018;59:1-9.

13. Arbury S, Hodgson M, Zankowski D, Lipscomb J. Workplace violence training programs for health care workers: an analysis of program elements. Workplace Health Saf 2017;65:266-72.

14. Medical Council of India. List of colleges teaching MBBS [homepage on the Internet]. New Delhi: MCl; 2018 [cited 
2020 Mar 19]. Available from: https://www.mciindia.org/CMS/ information-desk/for-students-to-study-in-india/ist-ofcollege-teaching-mbbs

15. Ranjan R, Meenakshi, Singh M, Pal R, Das JK, Gupta S. Epidemiology of violence against medical practitioners in a developing country (2006-2017). J Health Res Rev 2018;5: 153-60.

16. Singh G, Singh A, Chaturvedi S, Khan S. Workplace violence against resident doctors: a multicentric study from government medical colleges of Uttar Pradesh. Indian J Public Health 2019;63:143-6.

17. Kumar M, Verma M, Das T, Pardeshi G, Kishore J, Padmanandan A. A study of workplace violence experienced by doctors and associated risk factors in a tertiary care hospital of South Delhi, India. J Clin Diagn Res. 2016;10:LC06-10.

18. ILO, ICN, WHO, PSI. Joint programme on work place violence in health sector. Workplace violence in the health sector country case studies research instruments; survey questionnaire, English. Geneva: Publications Bureau International Labour Office; 2003.

19. Gohil RK, Singh PK, Saxena N, Patel G. Work place violence against resident doctors of a tertiary care hospital in Delhi, India. Int Surgery J 2019;6:975-81.

20. Anand T, Grover S, Kumar R, Kumar M, Ingle GK. Workplace violence against resident doctors in a tertiary care hospital in Delhi. Natl Med J India 2016;29:344-8.

21. Ori J, Devi NS, Singh AB, Thongam K, Padu J, Abhilesh R. Prevalence and attitude of workplace violence among the post graduate students in a tertiary hospital in Manipur. $J$ Med Soc 2014;28:25-8.

22. Lu L, Dong M, Wang SB, Zhang L, Ng CH, Ungvari GS et al. Prevalence of workplace violence against healthcare professionals in China: a comprehensive meta-analysis of observational surveys. Trauma Violence Abuse 2020; 21:498509.

23. Lanza ML, Zeiss RA, Rierdan J. Non-physical violence: a risk factor for physical violence in health care settings. AAOHN J 2006;54:397-402.

24. Boafo IM, Hancock P, Gringart E. Sources, incidence and effects of non-physical workplace violence against nurses in Ghana. Nurs Open 2016;3:99-109.

25. William Reddy. The management of violence against staff in the healthcare sector. In: European Agency for Safety and Health at Work. European week for safety and health at work 2002. Luxembourg: Office for Official Publications of the European Communities; 2002;p.47-50.

26. Morken $\mathrm{T}$, Johansen $\mathrm{IH}$, Alsaker K. Dealing with workplace violence in emergency primary health care: a focus group study. BMC Fam Pract 2015;16:1-7.

27. Shafran-Tikva S, Chinitz D, Stern Z, Feder-Bubis P. Violence against physicians and nurses in a hospital: How does it happen? A mixed-methods study. Isr J Health Policy Res 2017; $6: 59$.

28. Martinez AJS. Managing workplace violence with evidence based interventions: a literature review. J Psychosoc Nurs Ment Health Serv 2016;54:31-6. 